\title{
The Relationship Between the Circular Polarization and the Magnetic Field for Astrophysical Masers with Weak Zeeman Splitting
}

\author{
W. D. Watson and H. W. Wyld \\ Physics Department, University of Illinois at Urbana-Champaign, 1110 West Green Street, \\ Urbana, IL 61801-3080
}

\begin{abstract}
The relationship between the magnetic field and the circular polarization of astrophysical maser radiation due to the Zeeman effect under idealized conditions is investigated when the Zeeman splitting is much smaller than the spectral linebreadth and when radiative saturation is significant. The description of the circular polarization, as well as inferences about the magnetic field from the observations, are clearest when the rate for stimulated emission is much less than the Zeeman splitting. The calculations here are performed in this regime, which is relevant for some (if not most) observations of astrophysical masers. We demonstrate that Stokes- $V$ is proportional to the Zeeman splitting and the fractional linear polarization is independent of the Zeeman splitting when the ratio of the Zeeman splitting to the spectral line breadth is small-less than about 0.1 . In contrast to its behavior for ordinary spectral lines, the circular polarization for masers that are at least partially saturated does not decrease with increasing angle between the magnetic field and the line-of-sight until they are nearly perpendicular.
\end{abstract}

Subject headings: magnetic fields-masers-polarization

\section{INTRODUCTION}

The circular polarization of maser radiation where the Zeeman splitting is much smaller than the spectral linebreadth is utilized in efforts to obtain information about the magnetic field in various astronomical contexts (e.g., for recent work, Kemball \& Diamond 1997; Sarma, Troland, \& Romney 2001; Vlemmings, Diamond, \& van Langevelde 2001; Yusef-Zadeh et al. 1999). For a two-level, non-masing ("thermal") spectral line, a simple relationship exists

$$
V /(\partial I / \partial v)=p B \cos \theta
$$

that involves Stokes- $V$, the derivative of the intensity $I$ with respect to Doppler velocity $v$, the magnetic field $B$, and the angle $\theta$ between the magnetic field and the line-of-sight ( $p$ is a constant of the molecular physics for the specific transition). When maser saturation is unimportant, the relationship can readily be seen as applicable for masers as well (e.g., Fiebig \& Gusten 1989). 
Though maser polarization in the presence of saturation was the focus of the classic investigation of Goldreich, Keeley \& Kwan (1973; hereafter GKK), they provide no guidance about the circular polarization for weak Zeeman splitting. GKK only consider line center, where the circular polarization is zero in the weak splitting regime. For a summary of the theory of maser polarization, see Watson (2001).

The GKK idealization is a uniformly pumped, linear maser in steady-state. The seed radiation is external, weak continuum radiation and the masing involves an angular momentum $J=1-0$ transition in the presence of a constant magnetic field. Even though the actual circumstances may be more complicated, the idealization and solutions of GKK have served as the basis for discussions of maser polarization in general. In this Letter, we partially remedy the omission of GKK for weak splitting by calculating Stokes- $V$ for this basic idealization in the limit that the rate $R$ for stimulated emission is much less than the Zeeman splitting $g \Omega$ (where $\Omega=e B / m_{e} c$ and $g$ is the Lande $g$-factor). This limit certainly is applicable when $g \Omega$ is a noticeable fraction (greater than a few percent) of the linewidth as occurs for the $1720 \mathrm{MHz} O H$ masers. It probably also is relevant for the $22 \mathrm{GHz}$ water masers for which $g \Omega \simeq 10^{4} B(G) s^{-1}$ for the $F=7-6$ transition and provides at least a basis for discussion for the $\mathrm{SiO}$ masers where $g \Omega \simeq 10^{3} B(G) s^{-1}$. As a benchmark, the measure of the degree of saturation is the ratio of $R$ to the loss rate $\Gamma$ which ordinarily is taken to be about $1 \mathrm{~s}^{-1}$ and $5 \mathrm{~s}^{-1}$ for the $\mathrm{H}_{2} \mathrm{O}$ and $\mathrm{SiO}$ masers, respectively, and somewhat less for the $1720 \mathrm{MHz}$ masers. We limit our attention to $g \Omega \gg R$ because here the modifications to the standard Zeeman effect are moderate and the magnetic field can still be inferred from Stokes- $V$ with some confidence. Both the conceptualization and calculation of Stokes- $V$ are considerably simpler in this regime because only "ordinary rate equations" and "ordinary molecular populations" of the magnetic substates are involved. In contrast, when $g \Omega \gg R$ is not satisfied, Stokes- $V$ can be much larger (sometimes referred to as "non-Zeeman" effects) than expected from the standard Zeeman relationship (Nedoluha \& Watson 1994). It is unclear whether useful information about the magnetic field can be inferred from the observations in this regime. The more involved methods of the quantum mechanical density matrix must be utilized in this regime to incorporate the correlations between the magnetic substates. Large, non-Zeeman circular polarization can also be generated by effects that would not be present in the GKK idealization for masing, such as changes in the direction of the magnetic field within the masing region (Wiebe \& Watson 1998).

Calculations of the type being presented here have previously been performed (Nedoluha \& Watson 1992), but only for a quite limited range of saturation and angles $\theta$, and only for the molecular parameters that are specific to the $22 \mathrm{GHz}$ masing transition. That is, for the high angular momenta $(F=7,6,5, \& 4)$ and when the values of the magnetic moments are different for the upper and lower molecular states. These calculations did demonstrate that maser saturation alters equation (1) and that its effect is to increase Stokes- $V$ much more at large angles than at small angles relative to that expected from equation (1). The latter is especially significant because lines-of-sight to masers are often likely to be nearly perpendicular to the magnetic fields (e.g., toward circumstellar masing rings, toward the centers of accretion disks viewed edge-on). 
The intent of this Letter is to provide a comprehensive description of the relationship between the circular polarization and the magnetic field within the limitations outlined above, and to do this

for the idealized masing conditions treated by GKK which have served as a basis for discussing maser polarization.

\section{CALCULATIONS}

The rate equations for the normalized (dimensionless) population differences $n_{+}, n_{-}$, and $n_{0}$ between the magnetic substates $\mathrm{m}=+1,-1$, and 0 of the upper (angular momentum $J=1$ ) energy level and the $\mathrm{m}=0$ substate of the lower $(J=0)$ level, respectively, are functions of the molecular velocity $v$ and can be expressed by (e.g., Wallin \& Watson 1995; also GKK)

$$
1=\left(1+2 R_{+}\right) n_{+}+R_{0} n_{0}+R_{-} n_{-}
$$

and the two related equations that are obtained by exchanging the \pm and 0 indexes in equation (2). "Phenomenological" pumping and loss rates " $\Lambda$ " and " $\Gamma$ ", which are standard in maser theory, have been utilized and incorporated into the normalizations of the populations to obtain equation (2). For a unidirectional linear maser, the normalized rates for stimulated emission in equation (2) are

$$
R_{ \pm}=I_{ \pm}\left(1+\cos ^{2} \theta\right)+Q_{ \pm} \sin ^{2} \theta \pm 2 V_{ \pm} \cos \theta
$$

and

$$
R_{0}=2\left(I_{0}-Q_{0}\right) \sin ^{2} \theta
$$

where the subscripts \pm and 0 indicate that the intensities are to be evaluated at the frequencies (angular) $\omega_{ \pm}$and $\omega_{0}$ for which

$$
(1-v / c) \omega_{ \pm}=\omega_{R} \pm g \Omega / 2
$$

and

$$
(1-v / c) \omega_{0}=\omega_{R}
$$

Here, $\omega_{R}$ is the resonance frequency of the transition and $\pm g \Omega / 2$ are the Zeeman shifts of the $m=$ \pm 1 magnetic substates. The dimensionless Stokes intensities $(I, Q, V)$ are actual intensities divided by the characteristic saturation intensity $I_{S}=\left(8 \hbar \omega^{3} \Gamma / 3 \pi c^{2} A_{E}\right)$ where $A_{E}$ is the Einstein A-value 
for the transition. Note that whereas $R_{ \pm}$and $R_{0}$ are dimensionless, $R$ itself is not normalized and retains its usual dimensions $\left(\right.$ time $^{-1}$ ) in our discussions. The radiative transfer equations

$$
\begin{gathered}
d I / d s=A I+B Q+C V \\
d Q / d s=A Q+B I \\
d V / d s=A V+C I
\end{gathered}
$$

are at a specific frequency $\omega$ and can be solved numerically as a function of the normalized distance $s$ with the expressions

$$
\begin{gathered}
A=\left(1+\cos ^{2} \theta\right)\left(f_{+} n_{+}+f_{-} n_{-}\right)+2 f_{0} n_{0} \sin ^{2} \theta \\
B=\sin ^{2} \theta\left(f_{+} n_{+}+f_{-} n_{-}-2 f_{0} n_{0}\right) \\
C=2 \cos \theta\left(f_{+} n_{+}-f_{-} n_{-}\right)
\end{gathered}
$$

where the $f$ 's are Maxwellian distributions for the component of the velocity that is along the path of the radiation. In these equations, $f_{ \pm}, n_{ \pm}, f_{0}$ and $n_{0}$ are evaluated at velocities $v_{ \pm}$and $v_{0}$ given by $\left(1-v_{ \pm} / c\right) \omega=\omega_{R} \pm g \Omega / 2$ and $\left(1-v_{0} / c\right) \omega=\omega_{R}$.

Starting from the initial conditions $I \ll 1$ and $Q=0=V$, the radiative transfer equations are integrated numerically at a sufficient number of frequencies $\omega$ to delineate the profile of the spectral line. We emphasize that the foregoing equations are valid only when $g \Omega \gg R$. They are the same as would describe the polarization of ordinary thermal radiation - the only differences being the sign of the net pumping rate which is reflected in the sign of the " 1 " on the left-hand-side of equation (2) and the ignoring of spontaneous emission.

The main results are presented in the Figure 1 in terms of the ratios $V /(p B \partial I / \partial v)$ and $Q / I$ computed for a value of $g \Omega$ that is much less than the spectral linebreadth and at a frequency that is essentially (though not exactly) at line center. These ratios are useful because, in the weak splittng regime and consistent with equation (1), they tend to be independent of $g \Omega$ and constant with frequency across the spectral line. At frequencies where the bulk of the intensity occurs, we have confirmed that the ratios in Figure 1 are independent of frequency to a good approximation in the weak splitting regime as delineated in the following paragraph. Exceptions to this generalization do occur at the angles for which the deviations from equation (1) are greatest 
and also the intensities are in the neighborhood of $I=1$. Where there are variations in $V /(p B \partial I / \partial v)$ and $Q / I$ with frequency, these variations are modest and are smallest near line center. Note that the properties of the radiation at different frequencies within the spectral line are not completely independent because of the coupling that is indicated in equations (5) and (6), and in the analogous expressions for $v_{ \pm}$and $v_{0}$. In detail, the rate for stimulated emission depends on the polarization of the radiation, on the angle $\theta$, and is not exactly the same for all of the magnetic substates. It nevertheless is useful to utilize $I$ (which is normalized by the characteristic saturation intensity) as the measure of the degree of saturation. That is, $I \simeq R / \Gamma$.

The goal here is limited to providing information on the polarization characteristics for the limit of weak Zeeman splitting - that is, for small enough $g \Omega$ that the ratios in Figure 1 are independent of $g \Omega$ to a good approximation for the bulk of the radiation within the spectral line. The precise accuracy of this approximation for a specific $g \Omega$ depends upon angle $\theta$, the degree of saturation, the Doppler velocity within the spectral line, and is not exactly the same for the linear and the circular polarization. Nevertheless, some useful generalizations can be made. We compute polarizations for $g \Omega$ as large as one-fifth of the FWHM thermal Doppler breadth $\Delta \omega_{t}\left(\Delta \omega_{t}=2.4 \omega_{R}\left[k T / M c^{2}\right]^{1 / 2}\right)$. As long as the degree of saturation is greater than one, the ratios presented in the Figure 1 are independent of $g \Omega$ to an accuracy of a few percent. Likewise, as long as $g \Omega / \Delta \omega_{t} \leq 0.05$, these ratios also are independent of $g \Omega$ to a similar accuracy regardless of the degree of saturation. For lower saturations $-I$ down to $0.01-$ and $0.2 \geq g \Omega / \Delta \omega_{t} \geq 0.05$, the ratio $V /(p B \partial I / \partial v)$ is still independent of $g \Omega$ to within about twenty percent accuracy. The main deviations occur at angles less than about forty-five degrees where the effects of saturation are the least.

The main effect of saturation on the circular polarization is readily evident in the Figure 1. Instead of decreasing as $\cos \theta$ when the angle $\theta$ increases from zero, Stokes- $V$ tends to increase until a relatively large angle is reached whose value is determined by the exact degree of saturation. At $\theta=0$, Stokes- $V$ becomes equal to its value for unsaturated masing. The nearly discontinuous change reflects the change in the molecular populations that is associated with the similarly rapid variation of the linear polarization as $\theta$ approaches zero in the GKK theory. The variation of the linear polarization with saturation can be sensitive to the angular momentum of the molecular states (e.g., Deguchi \& Watson 1990). We have thus performed similar calculations for a $J=2-1$ masing transition, as well, with the idealization that $g$ is the same for the upper and lower energy levels (hence, $p=g e / 2 m_{e} \omega_{R}$ is the same for both transitions). These also are presented in Figure 1 , along with additional results from a further calculation in which the $J=1-0$ masing is treated as bidirectional. The optical depths are the same for radiation propagating in opposite directions in a linear maser. If the external seed radiation is similar at both ends of the maser (or if the seed radiation is due to spontaneous emission), the radiation propagating in the two directions will also be similar and the maser will be "bidirectional". It is known that the variation of the linear polarization with saturation can be different for uni- and bidirectional masers (Western \& Watson 1984). However, the comparisons in Figure 1 demonstrate that the differences for the circular polarization are small for both of these modifications which we have considered beyond the basic 
unidirectional $J=1-0$ maser .

The fractional linear polarization in Figure 1 is identical with that obtained in previous calculations. We present it here in the same format as the circular polarization for convenience in relating the two polarizations. At high saturation, the fractional linear polarizations in our calculations (see also Western \& Watson 1984) do reach those of GKK - which are obtained by GKK only for the limit of high saturation. The fractional linear polarization is seen in Figure 1 to increase more rapidly as a function of saturation for $J=1-0$ than for $J=2-1$ masers, and more rapidly for bidirectional than for unidirectional masers. As found previously, however, to reach the very highest $Q / I$ given by GKK, the degree of saturation must be implausibly high for $J=2-1$ masers. Anisotropic (or " $m$-dependent") pumping by starlight is thus the most likely cause for the highest fractional linear polarizations of the $\mathrm{SiO}$ and perhaps other masers (Western \& Watson 1983).

Unfortunately, the degree of radiative saturation of astrophysical masers is a longstanding uncertainty. In addition to the surface brightness of the maser, the saturation depends on the angle into which the radiation is beamed - a quantity for which direct estimates ordinarily are unreliable (e.g., Watson \& Wyld 2000). Note that the "intensities" of the linear maser formulation (by us and by GKK) actually are "mean intensities" $\times 4 \pi$. Hence, intensities from the observations must be multiplied by the solid angle of the beam (as well as divided by $I_{S}$ ) in order to relate them to the $I$ in the Figures. A comparison of the observed linear polarization with that in Figure 1 can be helpful to restrict the degree of saturation if there are no contaminating effects such as anisotropic pumping, multiple components, or Faraday rotation. The narrowing of the spectral line during unsaturated maser amplification, followed by rebroadening of the line when the maser becomes saturated, can also be used to obtain an indication of saturation (e.g., Nedoluha \& Watson 1991) if there are no velocity gradients or multiple components within the maser. A consideration of the linebreadths of certain prominent $22 \mathrm{GHz}$ water masers indicates that the influence of saturation on Stokes- $V$ probably is modest for these masers (Nedoluha \& Watson 1992). The linebreadth in our calculations is given in Figure 2 as a function of $I$. It does depend somewhat on the intensity of the incident continuum seed radiation. The computations in Figure 1 are performed for an incident intensity $I=10^{-9}$ which we believe to be representative. We have also performed computations analogous to those in Figure 1 when the incident intensity $I$ is $10^{-5}$ as might occur when the masing gas is amplifying a strong continuum source. The resulting $Q / I$ are essentially identical to those in Figure 1. The Stokes- $V$ also are quite similar to those in Figure 1, except near the peaks of the curves for the the largest $I\left(\gtrsim 10^{2}\right)$ where they are smaller by $15-20 \%$.

In summary, when the observed masers are believed to be at least somewhat saturated, but there is no good information about the angle $\theta$ nor about the exact degree of saturation, simply removing the $\cos \theta$ in equation (1) would seem to provide the best way at present to infer magnetic field strengths from the observed Stokes- $V$ in the weak splitting regime when "non-Zeeman effects" can be ignored. Saturation with $I \gtrsim 10^{2}$ (and probably even $I \gtrsim 10$ ) seems unlikely for astrophysical masers. In contrast to the linear polarization, the circular polarization is relatively insensitive to the angular momentum of the molecular states. We emphasize that our results are 
applicable in detail only for the idealized masing conditions on which the calculations are based (see Introduction). In addition to non-Zeeman effects, velocity gradients, anisotropic pumping, and multiple hyperfine components may be present, but are not considered here. For example, the $22 \mathrm{GHz}$ masing transition of water probably consists of multiple hyperfine components so that equation (1) is unlikely to be directly applicable for it (but see Nedoluha \& Watson 1992).

This research has been supported in part by NSF Grant AST99-88104

\section{REFERENCES}

Deguchi, S., \& Watson, W.D. 1990, ApJ, 354, 649

Fiebig, D., \& Gusten, R. 1989, A\&A, 214, 333

Goldreich, P., Keeley, D.S., \& Kwan, J.Y. 1973, ApJ, 179,111 [GKK]

Kemball, A. J., \& Diamond, P. J.. 1997, ApJ, 481, L111

Nedoluha, G. E., \& Watson, W. D. 1991, ApJ, 367, L63

Nedoluha, G. E., \& Watson, W. D. 1992, ApJ, 384, 185

Nedoluha, G. E., \& Watson, W. D. 1994, ApJ, 423, 394

Sarma, A. P., Troland, T. H., \& Romney, J. D. 2001, ApJ, 554, L217

Vlemmings, W., Diamond, P. J.,\& van Langevelde, H. J. 2001, in proceedings of IAU Symposium 206: Cosmic Masers, in press

Wallin, B.K., \& Watson, W.D. 1995, ApJ, 445, 465

Watson, W. D. 2001, in proceedings of IAU Symposium 206: Cosmic Masers, in press

Watson, W.D., \& Wyld, H. W. 2000, ApJ, 530, 207

Western, L.R., \& Watson, W. D. 1983, ApJ, 275, 195

Western, L.R., \& Watson, W. D. 1984, ApJ, 285, 158

Wiebe, D.S., \& Watson, W. D. 1998, ApJ, 503, L71

Yusef-Zadeh, F., Roberts, D. A., Goss, W. M., Frail, D. A., \& Green, A. J. 1999, ApJ, 512,230 
Fig. 1. - Circular and linear polarization of maser radiation as a function of the cosine of the angle $\theta$ between the direction of the magnetic field and the line of sight. Polarizations are presented for $J=1-0$ unidirectional and bidirectional masers, and for $J=2-1$ unidirectional masers, in separate panels as indicated by the label in each panel. The curves are labeled by the $\log _{10}$ of the intensity $I$ which is essentially the degree of saturation. Curves (some of which overlap) are plotted for $I=10^{-2}, 10^{-1}, 1,3,10,10^{2}, 10^{3}$, and $10^{4}$.

(upper three panels) The circular polarization as measured by the magnitude of $V /(p B \partial I / \partial v)$ which is equal to $\cos \theta$ in the unsaturated limit.

(lower three panels) The fractional linear polarization. Stokes- $Q$ is positive when the linear polarization is perpendicular to the direction of the magnetic field projected onto the plane of the sky.

Fig. 2.- The ratio of the spectral linebreadth (FWHM) of the maser radiation to the Doppler breadth $\Delta \omega_{t}$ (FWHM) of the thermal velocities of the masing molecules, as a function of the $\log _{10}$ of the intensity $I$. Linebreadths are shown for $J=1-0$ unidirectional (solid lines), $J=1-0$ bidirectional (dotted lines), $J=2-1$ unidirectional (dashed lines) masers with intensities for the incident continuum radiation of $I=10^{-5}$, as well as for $I=10^{-9}$ which we consider to be most representative. 


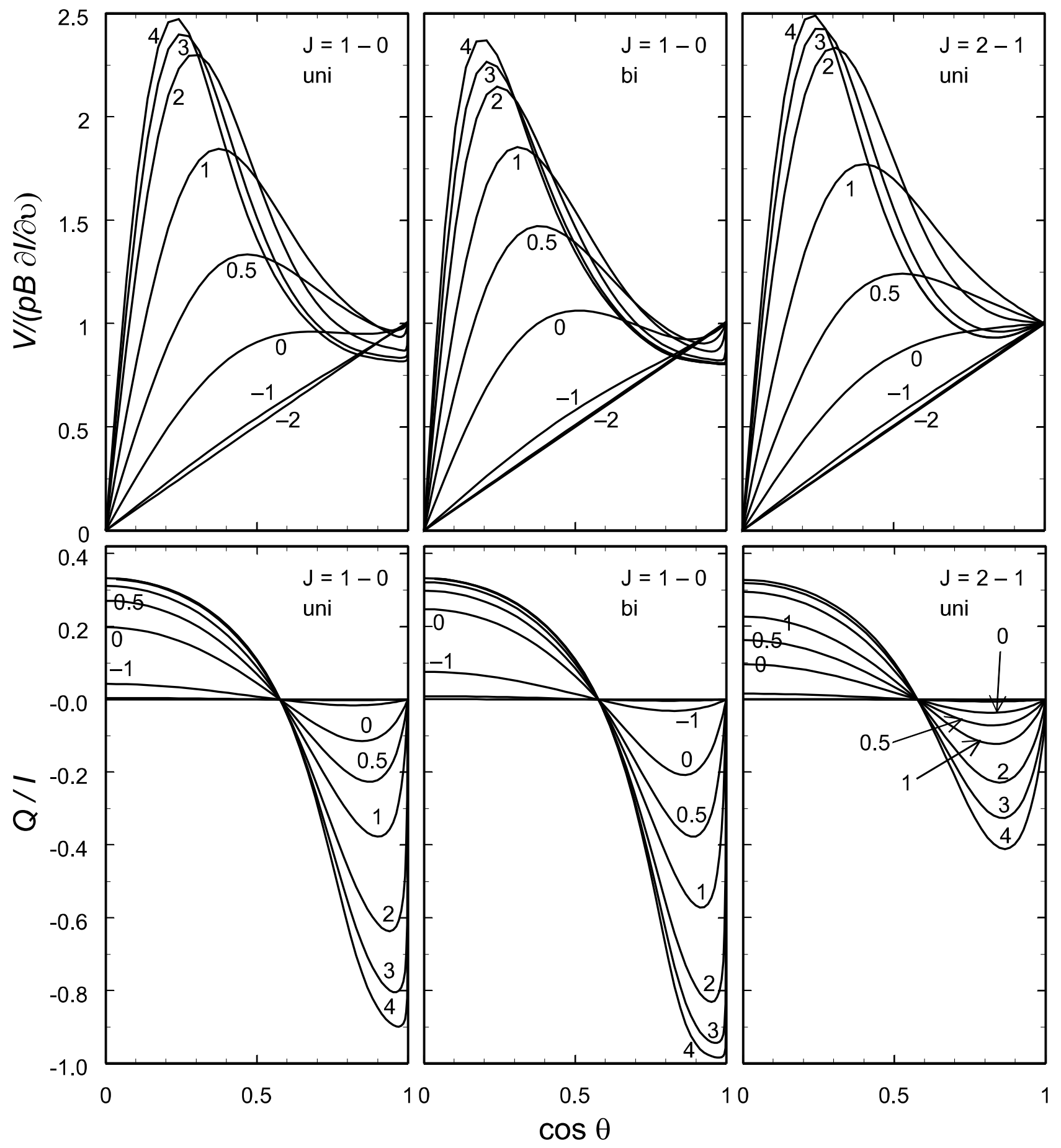




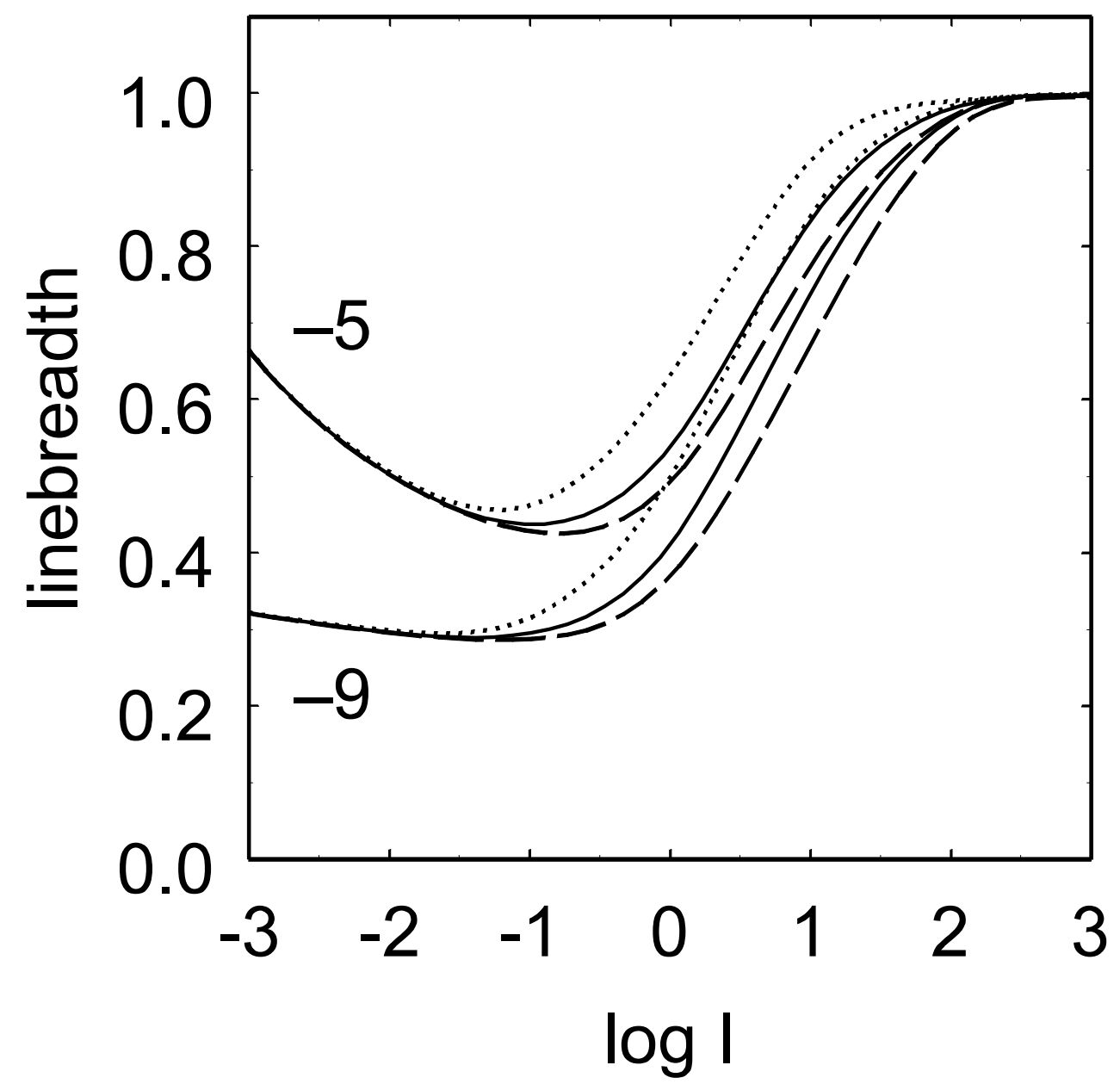

